Submitted 2007 OCtober 5; ACCePted 2008 February

Preprint typeset using $\mathrm{LAT}_{\mathrm{E} X} \mathrm{X}$ style emulateapj v. 04/21/05

\title{
STAR FORMATION NEAR PHOTODISSOCIATION REGIONS: DETECTION OF A PECULIAR PROTOSTAR NEAR CED 2011,2,3
}

\author{
Javier R. Goicoechea ${ }^{4}$, Olivier Berné ${ }^{5}$, Maryvonne Gerin ${ }^{4}$, Christine Joblin ${ }^{5}$, David Teyssier ${ }^{6}$ \\ ${ }^{4}$ LERMA-LRA, UMR 8112, CNRS, Observatoire de Paris et École Normale Supérieure, 24 rue Lhomond, 75231 Paris Cedex 05, France. \\ ${ }^{5}$ Centre d'Etude Spatiale des Rayonnements, CNRS et Université Paul Sabatier Toulouse 3, Observatoire Midi-Pyrénées, France. \\ and \\ ${ }^{6}$ European Space Astronomy Centre, ESAC, Urb. Villafranca del Castillo, P.O. Box 50727, Madrid 28080, Spain. \\ Submitted 2007 October 5; accepted 2008 February
}

\begin{abstract}
We present the detection and characterization of a peculiar low-mass protostar (IRAS 22129+7000) located $\sim 0.4 \mathrm{pc}$ from Cederblad 201 Photodissociation Region (PDR) and $\sim 0.2 \mathrm{pc}$ from the HH450 jet. The cold circumstellar envelope surrounding the object has been mapped through its $1.2 \mathrm{~mm}$ dust continuum emission with $I R A M-30 \mathrm{~m} / \mathrm{MAMBO}$. The deeply embedded protostar is clearly detected with Spitzer/MIPS $(70 \mu \mathrm{m})$, IRS $(20-35 \mu \mathrm{m})$ and IRAC $(4.5,5.8$, and $8 \mu \mathrm{m})$ but also in the $K_{s}$ band $(2.15 \mu \mathrm{m})$. Given the large near- and mid-IR excess in its spectral energy distribution, but large submillimeter-to-bolometric luminosity ratio $(\simeq 2 \%)$, IRAS $22129+7000$ must be a transition Class 0/I source and/or a multiple stellar system. Targeted observations of several molecular lines from CO, ${ }^{13} \mathrm{CO}, \mathrm{C}^{18} \mathrm{O}, \mathrm{HCO}^{+}$and $\mathrm{DCO}^{+}$have been obtained. The presence of a collimated molecular outflow mapped with the $C S O$ telescope in the $\mathrm{CO} J=3-2$ line suggests that the protostar/disk system is still accreting material from its natal envelope. Indeed, optically thick line profiles from high density tracers such as $\mathrm{HCO}^{+} J=1-0$ show a red-shifted-absorption asymmetry reminiscent of inward motions. We construct a preliminary physical model of the circumstellar envelope (including radial density and temperature gradients, velocity field and turbulence) that reproduces the observed line profiles and estimates the ionization fraction. The presence of both mechanical and (non-ionizing) FUV-radiative input makes the region an interesting case to study triggered star formation.
\end{abstract}

Subject headings: ISM : individual (IRAS 22129+7000; Ced 201; B175/L1219) — ISM : jets and outflows - stars: formation - stars: pre-main sequence

\section{INTRODUCTION}

Low-mass stars (sunlike; $\mathrm{M}_{\star}<8 M_{\odot}$ ) form from the gravitational collapse of dense molecular cores within giant molecular clouds. During the earliest stages of evolution, embryonic protostars are deeply embedded in cold and dusty envelopes of infalling material. In recent years, large millimeter $(\mathrm{mm})$ and submm continuum surveys have been carried out to detect the cold dust emission associated with these early birth-sites of star formation (André et al. 1993; Johnstone et al. 2000). During collapse, conservation of angular momentum combined with infall along the magnetic field lines leads to the formation of a rotating protoplanetary disk that drives the accretion process. At the same time, both mass and angular momentum are removed from the system by the onset of jets, collimated flows and the magnetic braking action (Bontemps et al. 1996; Cabrit et al. 2007). The resulting molecular outflow starts to erode and sweep up part of the natal envelope, contributing for the clearing of the circumstellar material and the termination of the infall phase (Arce \& Sargent 2006). The so-called Class 0 sources are deeply embedded protostars at their

\footnotetext{
${ }^{1}$ Based on observations obtained with the CSO telescope. The CSO telescope is operated by the California Institute of Technology under funding from the NSF, Grant No. AST-0540882.

2 Observations also obtained with IRAM telescopes, supported by INSU/CNRS (France), MPG (Germany), and IGN (Spain).

3 This work is based on observations made with the Spitzer Space Telescope, which is operated by the Jet Propulsion Laboratory, California Institute of Technology under a contract with NASA.
}

main accretion phase and represent the earliest type of young stellar objects (YSOs). Observationally they should show: $(i)$ Evidence of a central YSO (as revealed by the presence of a collimated outflow or an internal heating source); (ii) Centrally peaked but extended massive envelope (as traced by $\mathrm{mm}$ and submm dust continuum emission); (iii) High submm $(\lambda>350 \mu \mathrm{m})$ to bolometric luminosity ratio $\left(\mathrm{L}_{s m m} / \mathrm{L}_{b o l}>0.5 \%\right)$, and a spectral energy distribution (SED) similar to a single graybody at 15-30 K (André et al. 2000). On the other hand, Class I sources correspond to the late accretion phase (roughly half of the initial envelope mass is accreted in the Class 0 stage), they don't satisfy (iii) and can easily be detected at near-IR wavelengths (Lada 1999).

The detection of YSOs that do not exactly match the above canonical categories can provide the missing pieces of our current understanding of early disk evolution and early dissipation of the circumstellar envelope. These sources can thus potentially help to address, for example, how and when the velocity field around YSOs changes from infall to rotation dominated, which fraction of the initial mass envelope is dissipated and not accreted onto the star/disk, as well as the timescales for such dissipation (Brinch et al. 2007; Jørgensen et al. 2007)

In this paper we present the detection of a lowmass protostar in the Bok globule B175 (L1219) within the Cepheus Flare, a nearby $(\sim 400$ pc) star forming region $\sim 85 \mathrm{pc}$ above the galactic plane (Kun 1998; Nikolić \& Kun 2004). This cloud hosts the enigmatic Cederblad 201 reflection nebula (Wolf 1908; 

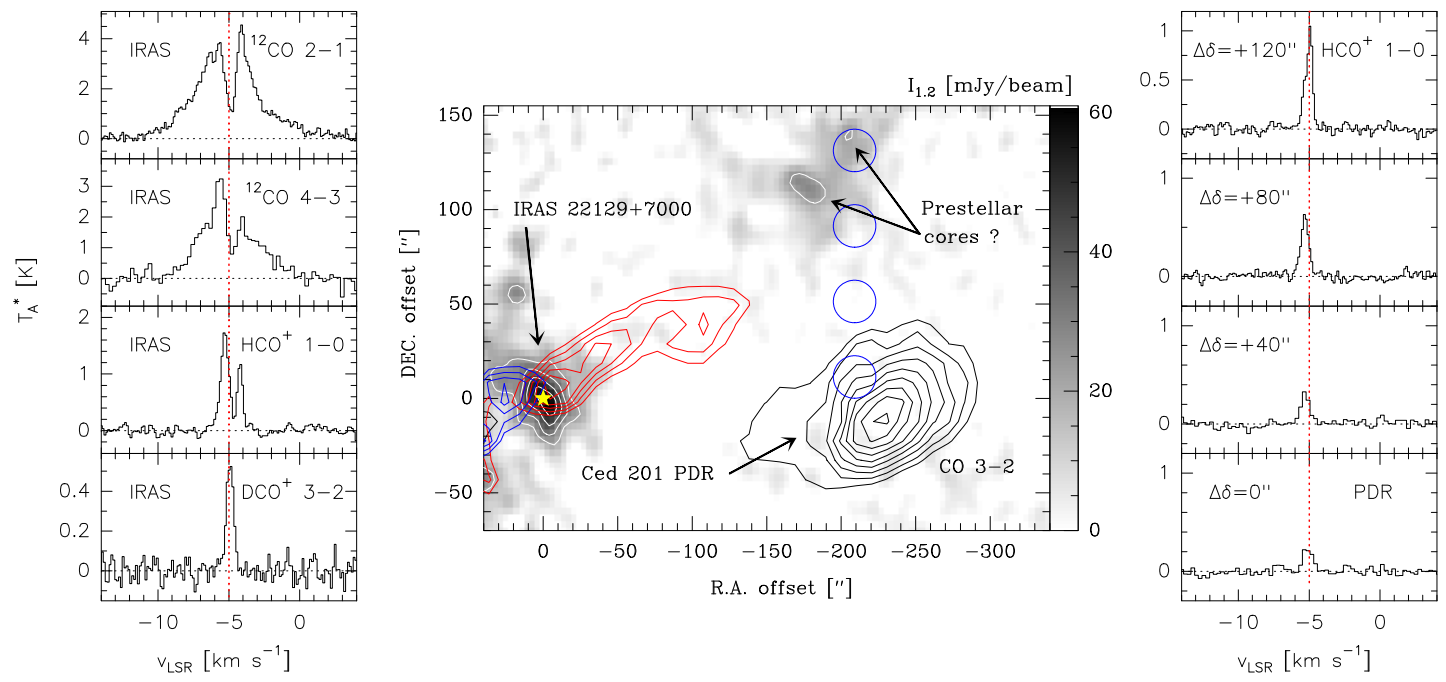

FIG. 1.- Middle panel: IRAM-30m/MAMBO $1.2 \mathrm{~mm}$-continuum emission of the region. The image is centered at IRAS $22129+7000$. White contours are displayed from $22(2 \sigma)$ to $55 \mathrm{mJy} / 15^{\prime \prime}$-beam in steps of $11 \mathrm{mJy} / 15^{\prime \prime}$-beam $(1 \sigma)$. The CO $J=3-2$ integrated intensity (from 5 to $9 \mathrm{~K} \mathrm{~km} \mathrm{~s}^{-1}$ in steps of $1 \mathrm{~K} \mathrm{~km} \mathrm{~s}^{-1}$ ) from the outflow is shown in red (from $v=-4$ to $-2 \mathrm{~km} \mathrm{~s}^{-1}$ ) and blue (from $v=-6$ to $-8 \mathrm{~km} \mathrm{~s}{ }^{-1}$ ). The integrated CO $J=3-2$ line intensity from Ced $201 \mathrm{PDR}\left(v=-5.5\right.$ to $-4.5 \mathrm{~km} \mathrm{~s}^{-1}$ ) is shown in black contours (from 6 to $20 \mathrm{~K} \mathrm{~km} \mathrm{~s}$ in steps of $\left.1 \mathrm{~K} \mathrm{~km} \mathrm{~s}^{-1}\right)$. Left panels: ${ }^{12} \mathrm{CO} J=2-1$ and $4-3, \mathrm{HCO}^{+} J=1-0$ and $\mathrm{DCO}^{+} J=3-2$ lines towards IRAS $22129+7000$ position. Right panels: Declination raster in the $\mathrm{HCO}^{+} J=1-0$ line. The vertical dashed line marks the rest velocity of the cloud $\left(-5 \mathrm{~km} \mathrm{~s}^{-1}\right)$.

Cesarsky et al. 2000), probably the result of a chance encounter with a $\mathrm{B} 9.5 \mathrm{~V}$ runaway star $\left(\mathrm{BD}+69^{\circ} 1231\right.$, $\mathrm{T}_{\text {eff }} \simeq 10,000 \mathrm{~K}$ ), which is moving through the cloud at $\sim 12 \mathrm{kms}^{-1}$ (Witt et al. 1987). The star is thus not a only a source of dissociating photons (the FUV radiation field is $\sim 200$ times the mean interstellar radiation field) but also a source of shocks and turbulence in the region. This interaction enables us to probe matter in a different situation than in classical PDRs in which the distance from the exciting star to the illuminated regions is large and/or in which the star was formed locally. In addition, the extended cloud hosts a Herbig-Haro jet (HH450), and several arsec-scale filaments of the supernova remnant (G110.3+11.3) that are rapidly approaching to the region (Bally \& Reipurth 2001). The presence of both mechanical (from shocks and outflows) and radiative input (from FUV photons) makes the region an interesting dynamics laboratory to study how star formation is proceeding through the cloud.

\section{OBSERVATIONS}

A $1.2 \mathrm{~mm}$ continuum emission map of the region was obtained at the IRAM-30m (Pico Veleta, Spain) in March 2005 using the 117-channel MAMBO bolometer array. The angular resolution is $\sim 11^{\prime \prime}$. A fast-mapping mode was used to map the region (Teyssier \& Sievers 1999). The total integration time was 1 hour, achieving a rms noise of $7 \mathrm{mJy} / 11^{\prime \prime}$-beam. Sky noise substraction and data analysis were carried out with the MOPSIC software (Zylka 1998). A $31^{\prime \prime} \times 22^{\prime \prime}$ fullwidth at half-maximum (FWHM) condensation with a peak intensity of $60 \pm 7 \mathrm{mJy} / 11^{\prime \prime}$-beam is detected $\sim 225^{\prime \prime}$ East of Ced $201 \mathrm{PDR}$. The integrated flux is $249 \pm 50 \mathrm{mJy}$, which reflects the estimated absolute calibration uncertainty. The resulting $1.2 \mathrm{~mm}$ emission map is shown in Figure 1 (smoothed to a beam resolution of $15^{\prime \prime}$ ).

The $\mathrm{HCO}^{+} J=1-0$ line was observed also at the $-30 \mathrm{~m}$ telescope during September 2005 using the B100 single sideband receiver with a channel resolution of $40 \mathrm{kHz}$. The main beam efficiency at $89 \mathrm{GHz}$ is 0.82 and the
TABLE 1

IRAS 22129+7000 PHOTOMETRIC DATA

\begin{tabular}{cccc}
\hline \hline $\begin{array}{c}\lambda \\
(\mu \mathrm{m})\end{array}$ & $\begin{array}{c}\text { Flux } \\
(\mathrm{Jy})\end{array}$ & Telescope & Aperture \\
& & & \\
\hline 1.25 & $\dagger 3.20 \times 10^{-4}$ & 2MASS & $3^{\prime \prime}$ \\
1.65 & $\dagger 3.60 \times 10^{-4}$ & 2MASS & $3^{\prime \prime}$ \\
2.15 & $3.00 \times 10^{-3}$ & 2MASS & $3^{\prime \prime}$ \\
4.5 & 0.17 & Spitzer/IRAC & $12.2^{\prime \prime}$ \\
5.8 & 0.38 & Spitzer/IRAC & $12.2^{\prime \prime}$ \\
8.0 & 0.31 & Spitzer/IRAC & $12.2^{\prime \prime}$ \\
12 & $\dagger 0.39$ & IRAS & $5^{\prime}$ \\
25 & 1.13 & IRAS & $5^{\prime}$ \\
60 & 3.46 & IRAS & $5^{\prime}$ \\
70 & 6.10 & Spitzer/MIPS & $1^{\prime}$ \\
100 & 16.00 & IRAS & $5^{\prime}$ \\
200 & 22.00 & KAO & $\sim 1^{\prime}$ \\
1200 & 0.25 & IRAM-30m/MAMBO & $31^{\prime \prime} \times 22^{\prime \prime *}$ \\
& & &
\end{tabular}

Note. — ${ }^{\dagger}$ Upper limit. ${ }^{*}$ Source FWHM size given.

angular resolution is $28^{\prime \prime}$. The line was observed towards the dust condensation and in a declination raster crossing Ced 201 PDR (see Fig. 1). Additional observations were performed at the CSO telescope (Mauna Kea, Hawaii) during July 2006 and June 2007. The telescope is equipped with SIS receivers operated in double sideband mode. The $\mathrm{CO} J=3-2$ map (Figs. 1 and 2) was made "on-the-fly". The $\mathrm{DCO}^{+} J=3-2$ line was mapped in a $\sim 40^{\prime \prime} \times 30^{\prime \prime}$ region around the dust condensation, whereas pointed observations in the CO $J=2-1$ and 4-3 lines were taken towards the dust emission peak (Fig. 1). The spectra were analyzed with a 1024 channel acoustooptic spectrometer with a total bandwidth of $50 \mathrm{MHz}$ and a resolution of $\sim 100 \mathrm{kHz}$. The main beam efficiencies of the telescope were $0.70,0.75$ and 0.53 at 230,345 , and $461 \mathrm{GHz}$ respectively. The angular resolution is $\sim 30^{\prime \prime}$ at $\mathrm{CO} J=2-1, \sim 20^{\prime \prime}$ at $\mathrm{CO} J=3-2$ and $\sim 15^{\prime \prime}$ at $\mathrm{CO} J=4-3$. Data was processed with GILDAS. 


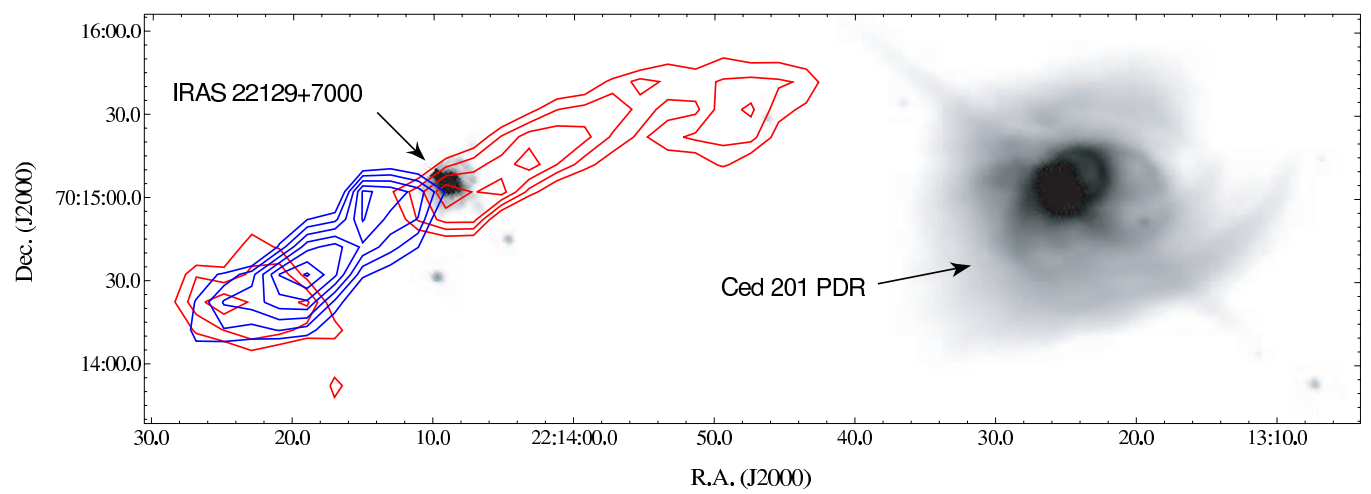

FIG. 2.- Spitzer/IRAC $8 \mu \mathrm{m}$ image of Ced201 region including the detection of IRAS $22129+7000$ protostar. The bright reflexion nebula (the PDR) is clearly seen at the west of the region. A well collimated outflow (as traced by CO $J=3-2$ high velocity line wing emission) emerges from the protostar position. Contours show the integrated intensity (from 5 to $9 \mathrm{~K} \mathrm{~km} \mathrm{~s}^{-1}$ in steps of $1 \mathrm{~K} \mathrm{~km} \mathrm{~s}^{-1}$ ) in the red (from $v=-4$ to $-2 \mathrm{~km} \mathrm{~s}^{-1}$ ) and blue (from $v=-6$ to $-8 \mathrm{~km} \mathrm{~s}^{-1}$ ) line wings respectively.

The Ced 201 region was also observed with Spitzer as part of our SPECPDR program ${ }^{4}$ (Joblin et al. 2005) with the Infrared Array Camera (IRAC) at 4.5, 5.8, and $8 \mu \mathrm{m}$ and with the Infrared Spectrograph (IRS). IRS data were obtained in the spectral mapping mode with the LL1 module (spectral range: 20 to $35 \mu \mathrm{m}$ ). The spectral cube of the source was assembled using the CUBISM software (Smith et al. 2007) from the Basic Calibrated Data (BCD) files. The 20 to $35 \mu \mathrm{m}$ data was obtained by integrating the cube spatially (see Berné et al. 2007). The $70 \mu \mathrm{m}$ Multiband Imaging Photometer for Spitzer (MIPS) data that are part of the PID 30790 program (Fazio et al.) were retrieved from the Spitzer archive. MIPS and IRAC post-BCD calibrated images were used directly without additional processing.

\section{RESULTS: DETECTION OF A YOUNG PROTOSTAR}

Figure 2 shows the detection of a compact continuum source at $\sim 8 \mu \mathrm{m}$ (warm dust) located $\sim 0.4$ pc East of Ced 201 PDR (Berné et al. 2007). The compact object is also detected at 4.5, 5.8, 20-35 and $70 \mu \mathrm{m}$ with Spitzer. The near-IR source position agrees with the continuum peak of the $1.2 \mathrm{~mm}$ extended emission (cold dust) detected in a larger field-of-view (Fig. 1). The best coordinates inferred from IRAC images are $\alpha_{2000}=22^{h} 14^{m} 08.3^{s}, \delta_{2000}=+70^{\circ} 15^{\prime} 06.7^{\prime \prime}$, which coincides with the IRAS $22129+7000$ source, clearly a YSO. A well collimated molecular outflow emanating from the object is seen along a SE-NW axis in the $\mathrm{CO} J=3-2$ line (Figs. 2 and 1). This outflow was previously detected in CO $J=1-0$ at lower angular resolution by Nikolić \& Kun (2004), who first suggested that IRAS 22129+7000 could be the driving source. We now detect the nearand mid-IR scattered light from the embedded protostar and the $\mathrm{mm}$ thermal emission from the surrounding dusty cocoon. In the following we examine the nature of this source and whether or not the presence of a PDR plays a role in the star formation process in the region.

\subsection{Peculiar SED Characteristics}

In order to investigate the emitted power in the different wavelength domains, the SED of IRAS $22129+7000$ has been built with our MAMBO and Spitzer observations and complemented with previous 2MASS

\footnotetext{
${ }^{4}$ http://www.cesr.fr/ joblin/SPECPDR_public/Home
}

$(2.15 \mu \mathrm{m})$, IRAS $(12,25,60$ and $100 \mu \mathrm{m})$ and $\mathrm{KAO}$ $(200 \mu \mathrm{m}$; Casey 1991) detections (Table 1). We fitted the SED with two graybodies, $B_{\lambda}(T)\left(1-e^{-\tau_{\lambda}}\right) \Omega$, where $\Omega$ is the solid angle subtended by the emitting region. In the far-IR and $\mathrm{mm}$ domain $\tau_{\lambda} \propto$ $\kappa_{1200}(1200 / \lambda)^{\beta}$ and $\mathrm{T}$ can be associated with the dust opacity and color temperature $\left(\simeq \mathrm{T}_{d}\right)$ of the extended envelope. Here we take a dust opacity (per gas+dust mass column density) of $\kappa_{1200}=0.01 \mathrm{~cm}^{2} \mathrm{~g}^{-1}$ at $1200 \mu \mathrm{m}$, the usual value adopted for the dust in protostellar envelopes (André et al. 2000). Because of the implicitly assumed grain growth and ice mantle formation (Ossenkopf \& Henning 1994), this value is a factor $\sim 5$ larger than $\kappa_{1200}$ for standard interstellar grains. $\Omega$ is fixed as the FWHM size of the envelope inferred from the $1.2 \mathrm{~mm}$ continuum map, while the column density of material is varied between $A_{V} \simeq 5$ and 30. Satisfactory fits are obtained for $\mathrm{T}_{d}=24-19 \mathrm{~K}$ and dust spectral indexes $\beta=1.4-1.8$ respectively (see the best fit as continuous blue curve in Fig. 3). A luminosity of $\mathrm{L}_{b o l}=5.5 \pm 0.5 L_{\odot}$ and a "bolometric temperature" of $\mathrm{T}_{b o l} \simeq 175 \mathrm{~K}$ (the temperature of a blackbody with the same SED mean frequency; Myers \& Ladd 1993) are inferred from the full fit.

In a first inspection, the resulting SED (Fig. 3) does not look like a typical Class 0 source in the near- and mid-IR (see e.g., Whitney et al. 2003). The improved sensitivity of the IR instrumentation compared to that available in the 90's has allowed the detection of scattered light from several Class 0 sources below $\sim 10 \mu \mathrm{m}$ (Tobin et al. 2007). In addition, the $\mathrm{K}_{s}$-band emission due to scattered light observed through a cavity opened by the outflow (with a favorable inclination angle) and/or $\mathrm{H}_{2}$ rovibrational emission from the outflow base, has also been detected in a few Class 0 sources (e.g., Tachihara et al. 2007). IRAS $22129+7000$ shows these observable characteristics but it also displays an intriguing strong, and rather flat, near- and mid-IR emission (as shown by the relatively high $\left.\mathrm{T}_{b o l}\right)$. In fact, Spitzer fluxes towards IRAS $22129+7000$ are consistent with the typical values observed in Class I sources with the same luminosity (Furlan et al. 2008), but an order of magnitude higher than in Class 0 sources (Tobin et al. 2007). On the other hand, IRAS $22129+7000$ shows a very large fraction of submm $(\lambda>350 \mu \mathrm{m})$ to bolometric luminosity $(\simeq 2 \%$; a signature of younger Class 0 sources still surrounded by massive dusty envelopes). 


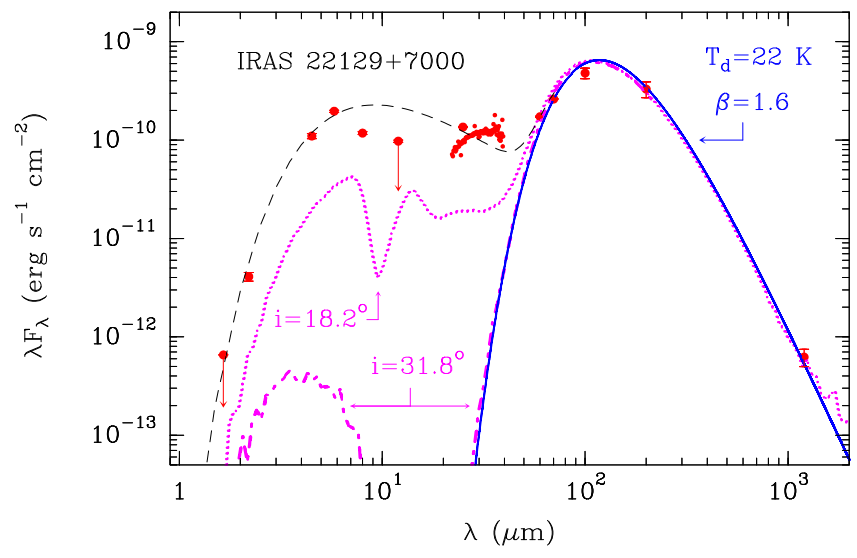

FIG. 3.- SED of IRAS $22129+7000$ combining data in the $\mathrm{H}$ and $K_{s}$ bands (2MASS; Skrutskie et al. 2006); $4.5,5.8$ and $8 \mu \mathrm{m}$ (Spitzer/IRAC); 20-35 $\mu \mathrm{m}$ (Spitzer/IRS, smaller points), 12, 25, 60 and $100 \mu \mathrm{m}$ (IRAS), $70 \mu \mathrm{m}$ (Spitzer/MIPS), $200 \mu \mathrm{m}$ (KAO: Casey 1991) and $1200 \mu \mathrm{m}$ (MAMBO). The Spitzer/IRS fluxes towards the source agree with those measured by IRAS at $25 \mu \mathrm{m}$ probing the compact nature of the mid-IR emission. The dashed curve is a simple fit using two graybodies with different temperatures. The continuous blue curve represents the circumstellar envelope with $\mathrm{T}_{d}=22 \mathrm{~K}$ and a spectral index of 1.6. The magenta pointed- and pointed-dashed curves show the best $2 \mathrm{D}$ radiative transfer models obtained with the Robitaille et al. (2007) fitter for two different inclinations $(i=0$ corresponds to "pole-on" and $i=90$ to "edgeon" configurations).

In an attempt to better understand the peculiar IR shape we tried to fit the SED with the $2 \mathrm{D}$ radiative transfer tool by Robitaille et al. (2007). The best models fitting the data are shown in Fig. 3 for two different inclination angles. While the long-wavelength emission from the circumstellar envelope is well reproduced by the models (and is almost not affected by the viewing angle), the short-wavelength emission from the protostellar/disk system is clearly underestimated and strongly depends on inclination. Therefore, a small viewing angle favors the detection of near- and mid-IR scattered light from the embedded protostar through the cavities cleared by the outflow. However, the blue- and red-shifted lobes of the CO outflow (Fig. 2) appear relatively well separated, and thus the source can not be completely "polein" (Cabrit \& Bertout 1986). In summary, the observed SED is peculiar, with a near-and mid-IR excess that can not be fully explained in terms of inclination effects.

\subsection{Circumstellar Envelope: Radial Analysis}

The cold dust surrounding the YSO has been mapped through its $1.2 \mathrm{~mm}$ emission (Fig. 1). As mm continuum emission is generally optically thin, the measured fluxes are proportional to the temperature--weighted mass content assuming that dust grains properties are known. In order to trace the mass distribution in the envelope, the radial intensity profile $I(r)$ has been analyzed by averaging the $1.2 \mathrm{~mm}$ fluxes within equidistant circular annulus (Fig. 4). An envelope radius of $R_{\text {out }} \simeq 15,000 \mathrm{AU}$ is inferred. Assuming spherical symmetry, the intensity profile $I(r) \propto r^{-m}$ contains information on the density distribution, $\rho(r) \propto r^{-p}$, and temperature distribution, $T_{d}(r) \propto r^{-q}$, with $p=m+1-q$ (e.g., Adams 1991).

Before convolving the expected intensity profile with the bolometer angular resolution, we divide the envelope in two regions (inner and outer) and try to fit the
TABLE 2

IRAS 22129+700: SED PARAMETERS

\begin{tabular}{|c|c|c|c|c|}
\hline $\begin{array}{l}\mathrm{L}_{b o l} \\
\left(\mathrm{~L}_{\odot}\right)\end{array}$ & $\begin{array}{l}\mathrm{T}_{b o l} \\
(\mathrm{~K})\end{array}$ & $\begin{array}{l}\mathrm{M}_{\text {env }} \\
\left(\mathrm{M}_{\odot}\right)\end{array}$ & $\begin{array}{c}\mathrm{L}_{s m m} / \mathrm{L}_{b o l} \\
(\%)^{c}\end{array}$ & $\begin{array}{c}\mathrm{M}_{e n v} / \mathrm{L}_{b o l} \\
\left(\mathrm{M}_{\odot} / \mathrm{L}_{\odot}\right)\end{array}$ \\
\hline $5.5 \pm 0.5$ & $\sim 175$ & $0.2^{a}-0.7^{b}$ & $\sim 2$ & $0.04^{a}-0.13^{b}$ \\
\hline
\end{tabular}

observed $1.2 \mathrm{~mm}$ emission profile with two power-laws. Fig. 4 shows that the dust emission in the outer envelope ( $r \gtrsim 5,000 \mathrm{AU} \gtrsim$ one beam) follows a very steep profile, with $m \simeq 1.9$, connecting the envelope with the lower density ambient cloud at $r \geq R_{\text {out }}$. Since the outer envelope angular radii are larger than the beam's FWHM, the convolution does not modify the slope of the intensity profile (e.g., Adams 1991; Motte \& André 2001). For the same reason, our observations can not resolve accurately the slope of the inner envelope $\left(r<R_{i n} \simeq 5,000 \mathrm{AU}\right)$. We take $m \lesssim 0.5$ as the expected value in a free-falling envelope. The line radiative transfer models shown in Sect. 3.4 are not inconsistent with this assumption.

Taking again a dust opacity of $\kappa_{1200}=0.01 \mathrm{~cm}^{2} \mathrm{~g}^{-1}$ and $\mathrm{T}_{d}=20 \mathrm{~K}$ (consistent with the SED analysis) we compute a total circumstellar mass of $\mathrm{M}_{e n v}=0.7 M_{\odot}$ within $r \leq R_{\text {out }}$. However, it is unlikely that all this mass can be finally accreted by the protostar/disk. First because the outflow will disperse a large fraction of it, and second, because differential rotation in the envelope is expected to decouple the inner rapidly infalling and rotating layers from the slowly collapsing and rotating outer regions (Belloche et al. 2002). Hence, we also computed $\mathrm{M}_{e n v}^{i n}=0.2 M_{\odot}$, the mass within one $11^{\prime \prime}$-beam $\left(r \lesssim \mathrm{R}_{i n}\right)$ as representative of the mass inside the inner envelope.

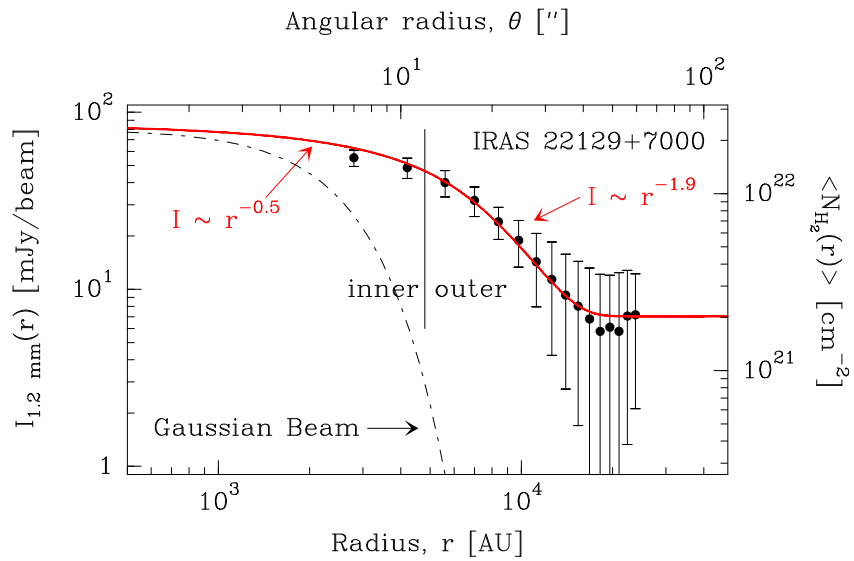

FIG. 4. $-1.2 \mathrm{~mm}$ radial intensity profile around IRAS $22129+7000$. The intensity (in mJy $/ 11^{\prime \prime}$-beam) has been averaged over circular annulus in steps of $3.5^{\prime \prime}$. The error bars show the standard deviation within each annulus. The dashed line represent a Gaussian beam with a FWHM of $11^{\prime \prime}$. The right axis shows an estimate of the $\mathrm{H}_{2}$ column density assuming $\kappa_{1.2}=0.01 \mathrm{~cm}^{2} \mathrm{~g}^{-1}$ and $\mathrm{T}_{d}=20 \mathrm{~K}$. The red curve shows a fit to the data including 2 intensity power-law profiles (convolved with the beam), with $I(r) \propto r^{-1.9}$ for $r \gtrsim 5,000 \mathrm{AU}$ and $I(r) \propto r^{-0.5}$ for $r \lesssim 5,000 \mathrm{AU}$. 
The steep slope of the $1.2 \mathrm{~mm}$ intensity profile suggests that a temperature gradient is also present. In particular, the dust thermal profile through the inner envelope should be dominated by accretion heating from the protostar/disk system with (Terebey et al. 1993):

$$
T_{d}(r) \approx 38 \mathrm{~K}\left(\frac{r}{100 \mathrm{AU}}\right)^{-q}\left(\frac{\mathrm{L}_{\star}}{\mathrm{L}_{\odot}}\right)^{q / 2}
$$

where $q$ depends on the dust grain properties through $q=2 /(4+\beta)$. Given the proximity of Ced $201 \mathrm{PDR}$, FUV photons from $\mathrm{BD}+69^{\circ} 1231$ star also heat the outer layers of IRAS 22129+7000 envelope. Penetration of this external FUV radiation field into the envelope greatly depends on the scattering properties of the dust particles, with larger penetration depths as grains grow toward bigger grains (Goicoechea \& Le Bourlot 2007). In the following we adopt a temperature profile that follows Eq. (1), reaches a minimum of $\sim 10 \mathrm{~K}$ at $r \sim 10,000 \mathrm{AU}$ and then increases up to $\simeq 15 \mathrm{~K}$ at $R_{\text {out }}$ due to external FUV heating (this temperature is consistent with our analysis of $\mathrm{CO}$ lines in the ambient cloud). Taking $q=0.36$ (using $\beta=1.6$ from the SED) and assuming $L_{\star} \simeq L_{b o l}$, the estimated mass of the envelope increases by a factor $\sim 2$ respect to the isothermal case because $T_{d}(r)$ drops below $20 \mathrm{~K}$ in a large fraction of the envelope. This difference adds to the dominant source of uncertainty in the mass determination, the dust composition and associated opacity.

Taking into account these caveats, the observed $1.2 \mathrm{~mm}$ intensity profile in the outer envelope is equivalent to a steep density profile $\rho\left(r>R_{i n}\right) \propto r^{-2.54}$ with a number density of $n\left(\mathrm{H}_{2}\right) \simeq(1-2) \times 10^{5} \mathrm{~cm}^{-3}$ at $R_{i n}$. Under these assumptions, the (beam averaged) extinction towards the peak (e.g., Motte \& André 2001) is $A_{V} \simeq 20-30$, a factor $\sim 10$ larger than the value implied by our $1.2 \mathrm{~mm}$ continuum observations in the ambient cloud.

\subsection{Gas Kinematics: Outflow and Infall?}

Figure 2 shows the outflow emanating from IRAS $22129+7000$ and extending $\sim 0.3 \mathrm{pc}$ in the NW direction (redshifted lobe) and $\sim 0.2 \mathrm{pc}$ in the SE direction (blueshifted lobe). In addition, IRAS $22129+7000$ is the driving source of the Herbig Haro HH450 jet detected $\sim 0.2$ pc SE from the object (Bally \& Reipurth 2001). The CO outflow is well collimated, with an aspect ratio of $\sim 3-4$ (the projected FWHM flow length/width ratio). CO $J=2-1$ to $4-3$ line profiles show high velocity wing emission from accelerated gas. Typical full line widths are consistent with an expansion velocity of $v_{\text {exp }} \simeq 7 \mathrm{~km} \mathrm{~s}^{-1}$ (Fig. 1). We thus estimate that the dynamical age of the outflow ( length $/ v_{\text {exp }}$ ) is $\sim 35,000 \mathrm{yr}$ (up to $\sim 10^{5} \mathrm{yr}$ if the inclination angle is $i=18^{\circ}$ ). The presence of a molecular outflow suggests that the protostar/disk system is still accreting material from the natal envelope. The high density tracer $\mathrm{HCO}^{+} J=1-0$ line, with a critical density of $n_{\mathrm{cr}}^{1-0} \simeq 2 \times 10^{5} \mathrm{~cm}^{-3}$, shows a deep red-shifted-absorption asymmetry in its optically thick line profile (when $n\left(H_{2}\right)>n_{\mathrm{cr}}^{J-J^{\prime}}$ the associated $J$ level is predominantly populated by collisions with $\mathrm{H}_{2}$ molecules and $\mathrm{T}_{e x}$, the transition excitation temperature, tends to the kinetic temperature). The depth and velocity position of the absorption dip are due to a decrease of $\mathrm{T}_{e x}$ at the outer edges of a (likely) infalling envelope (left insets in Fig. 1). As low- $J$ CO lines easily thermalize at lower densities $\left(n_{\mathrm{cr}} \simeq 10^{3-4} \mathrm{~cm}^{-3}\right)$, they may not be reliable tracers of the presence of infall motions but of the varying physical conditions in the beam. Among the observed CO lines, only the $J=4-3$ line starts to trace denser $\left(n_{\mathrm{cr}}^{4-3} \simeq 5 \times 10^{4} \mathrm{~cm}^{-3}\right)$, possibly infalling, gas. In anycase, the $\mathrm{HCO}^{+} J=1-0$ self-absorption dip is redshifted $0.3 \mathrm{~km} \mathrm{~s}^{-1}$ from the rest velocity of the cloud $\left(-5 \mathrm{~km} \mathrm{~s}^{-1}\right)$ and is not observed at nearby positions of the cloud where the line shows a pure Gaussian emission profile (right insets in Fig. 1). Although higher- $J$ optically thick lines from abundant high dipole moment molecules should be mapped to draw definitive conclusions, in the following we assume that the observed deep $\mathrm{HCO}^{+}$self-absorption is produced by inward motions.

The densest gas in the envelope has been traced with the $\mathrm{DCO}^{+} J=3-2$ line $\left(n_{\mathrm{cr}}^{3-2} \simeq 2 \times 10^{6} \mathrm{~cm}^{-3}\right)$ which shows compact $\left(\lesssim 40^{\prime \prime}\right)$ emission around IRAS $22129+7000$. Compared to the broad line wings from the $\mathrm{CO}$ outflow, the $\mathrm{DCO}^{+} J=3-2$ line shows the narrowest observed linewidth $\left(\Delta v_{\mathrm{FHWM}}=0.60 \pm 0.04 \mathrm{~km} \mathrm{~s}^{-1}\right)$. On the other hand, we used the $\mathrm{C}^{18} \mathrm{O} J=1-0$ line to trace lower density gas (e.g., the outer envelope). This line displays a broader linewidth $\left(\Delta v_{\mathrm{FHWM}}=0.96 \pm 0.02 \mathrm{~km} \mathrm{~s}^{-1}\right)$. The radiative transfer calculations presented in the next section confirm that both lines are optically thin and thus they are not broaden by line saturation. Therefore, the different velocity dispersions reflect the distinct regions where both line profiles are formed. Assuming $\mathrm{T}_{k}=10$ to $20 \mathrm{~K}$, the observed $\mathrm{DCO}^{+}$linewidths are 5 to 3.5 times larger than the expected thermal broadening. The required non-thermal velocity dispersion to fit the observed $\mathrm{DCO}^{+} J=3-2$ linewidth (from turbulence, infall or rotation) is $\sigma_{n t h} \simeq 0.25 \mathrm{~km} \mathrm{~s}^{-1}$ (with $\left.\Delta v_{\mathrm{FHWM}}=2.355 \times \sigma\right)$.

\subsection{Line Radiative Transfer Analysis}

In order to reproduce the observed molecular lines with a realistic model of the envelope, we have used a nonlocal and non-LTE excitation and radiative transfer code (Goicoechea et al. 2006). The code models an infalling spherical envelope and accounts for line trapping, collisional excitation ${ }^{5}$, and radiative excitation. Arbitrary velocity fields as well as density, temperature, and abundance radial gradients are included as input parameters. This is done by numerical discretization of the envelope in spherical shells. The nonlocal radiative transfer problem is then simulated by the emission of a determined number of model photons (cosmic mm background, continuum and line photons) using the Monte Carlo approach (Bernes 1979). The steady state statistical equilibrium equations are then solved iteratively and the nonLTE level populations are determined in the shells. The emergent line intensities along each line of sight are finally convolved with the telescope angular resolution.

\subsubsection{A First Model for the Circumstellar Envelope}

As our first attempt to understand IRAS 22129+7000 physical structure, we have modelled a spherical envelope

${ }^{5} \mathrm{CO}$ (and isotopologues) rotationally inelastic collisional rates have been derived from those of Flower (2001). $\mathrm{HCO}^{+}$and $\mathrm{DCO}^{+}$ collisional rates have been derived from those of Flower (1999). 


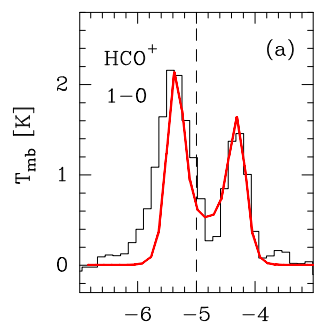

$\mathrm{V}_{\mathrm{LSR}}\left[\mathrm{km} \mathrm{s}^{-1}\right]$

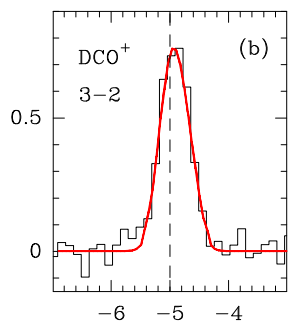

$\mathrm{v}_{\mathrm{LSR}}\left[\mathrm{km} \mathrm{s}^{-1}\right]$

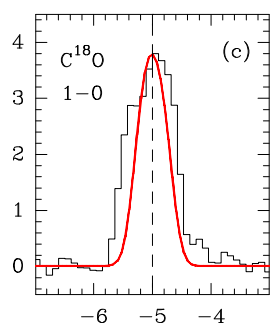

$\mathrm{v}_{\mathrm{LSR}}\left[\mathrm{km} \mathrm{s}^{-1}\right]$

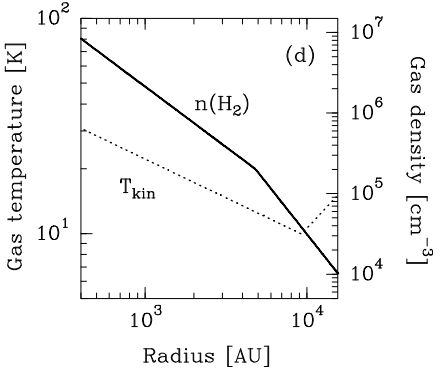

Radius [AU]

FIG. 5. - (a) $\mathrm{HCO}^{+} J=1-0,(b) \mathrm{DCO}^{+} J=3-2$ and $(c) \mathrm{C}^{18} \mathrm{O} J=1-0$ observations (histograms) towards IRAS $22129+7000$. Best radiative transfer models for the circumstellar envelope are also shown (red curves). Predicted line profiles have been convolved with the telescope angular resolution at each frequency. Intensity scale is in main beam temperature. (d) Density and temperature profiles used in the model.

with a radius $R_{\text {out }}=15,000 \mathrm{AU}$. We take the density and temperature profiles $\left(\rho(r) \propto r^{-2.54}\right.$ and $T(r) \propto r^{-0.34}$ plus external FUV heating) derived in the previous section for the outer envelope $\left(r>R_{i n} \simeq 5,000 \mathrm{AU}\right)$. Given the high densities expected in most of the envelope, we assume that gas and dust are thermalized (i.e., $T_{k}=T_{d}$ ).

Using the above density and temperature structure we run the model globally for $\mathrm{HCO}^{+}, \mathrm{DCO}^{+}$and $\mathrm{C}^{18} \mathrm{O}$ and try to find iteratively the best velocity fields and abundances fitting the observed line profiles. In order to reproduce the observed velocity of the CO $J=4$ 3 and $\mathrm{HCO}^{+} J=1-0$ redshifted absorption dips $(0.2$ and $0.3 \mathrm{~km} \mathrm{~s}^{-1}$ ) we adopt a uniform infalling velocity of $v_{\text {inf }}=0.2 \mathrm{~km} \mathrm{~s}^{-1}$ in the outer envelope. For $r \leq R_{\text {in }}$ (the inner envelope) we assume $\rho(r) \propto r^{-1.5}$, and $v_{\text {inf }}(r)=$ $0.2\left(r / R_{i n}\right)^{-0.5}$ i.e., the density and velocity profiles in a freefall inside-out collapsing envelope (Shu et al. 1987). Numerical simulations of collapse induced by external compression waves also predict those approximate $\rho(r)$ and $v_{\text {inf }}(r)$ profiles behind the expanding collapsewavefront (e.g., Hennebelle et al. 2003). These two regimes of infall (freefall and uniform) have been inferred in other YSOs with clear evidences of collapse (Belloche et al. 2002). Under the above conditions, $\mathrm{DCO}^{+}$optically thin lines constrain the remaining nonthermal broadening (turbulence in the following). Satisfactory fits to the $\mathrm{DCO}^{+} J=3-2$ line (with $\left[\mathrm{DCO}^{+} / \mathrm{H}_{2}\right]=(1-$ $2) \times 10^{-10}$ ) are obtained for $\sigma_{\text {turb }}=0.15 \mathrm{~km} \mathrm{~s}^{-1}$ (the turbulence on the size scale of the beam). Therefore, $\sigma_{\text {turb }}$ in the densest parts of the envelope (as traced by the $\mathrm{DCO}^{+}$) is subsonic, and probably a vestige of the low degree of turbulence found in prestellar cores (Bergin \& Tafalla 2007). Hereafter we adopt a uniform $\sigma_{\text {turb }}$ value of $0.15 \mathrm{~km} \mathrm{~s}^{-1}$ in the models of the envelope.

In order to reproduce the $\mathrm{HCO}^{+} J=1-0$ line profile we have added the contribution from the lower density (more turbulent) ambient cloud surrounding IRAS $22129+7000$ and producing a line-of-sight extinction of $\mathrm{A}_{V}=2-3$ (from $1.2 \mathrm{~mm}$ observations). Given the high $\mathrm{HCO}^{+}$dipole moment and large abundance (producing subthermal excitation and large opacities), low$J$ line photons from the dense envelope will be greatly attenuated and scattered over large areas by this low density halo (Cernicharo \& Guélin 1987). Note that the negligible $\mathrm{DCO}^{+}$and $\mathrm{C}^{18} \mathrm{O}$ column densities in the ambient cloud do not alter the $\mathrm{DCO}^{+}$and $\mathrm{C}^{18} \mathrm{O}$ line emission from the envelope itself. The density and temperature in the ambient cloud can be constrained with the observed CO lines. They are reproduced with $\mathrm{n}\left(\mathrm{H}_{2}\right) \simeq 1000 \mathrm{~cm}^{-3}$ and $\mathrm{T}_{k} \simeq 15 \mathrm{~K}$, in agreement with estimations by Witt et al. (1987). The halo ambient cloud in the model is at rest $\left(v_{\text {inf }}=0 \mathrm{~km} \mathrm{~s}^{-1}\right)$ but the broader observed linewidths require $\sigma_{\text {turb }}=0.30 \mathrm{~km} \mathrm{~s}^{-1}$ (supersonic), at least a factor 2 larger than in the circumstellar envelope. Figure 5 shows the best fits and the density and temperaure gradients used in the model. In spite of the good agreement, the model slightly underestimates the observed $\mathrm{HCO}^{+}$and $\mathrm{C}^{18} \mathrm{O} J=1-0$ linewidths, likely due to a minor contribution from the high velocity outflow (not modelled here).

The resulting large $\mathrm{DCO}^{+} / \mathrm{HCO}^{+}$abundance ratio $(\sim 0.02)$ found in IRAS $22129+7000$ is similar to that derived in cold and dense prestellar condensations near other PDRs (e.g., Pety et al. 2007). This is consistent for a species that shows enhanced abundance in the cold FUV-shielded gas (low ionization degree) affected by ongoing deuterium fractionation (where dissociative recombinations with electrons are its main destruction route). As a consequence, the $\mathrm{DCO}^{+} / \mathrm{HCO}^{+}$abundance ratio is roughly inversely proportional to the electrons abundance and we can estimate its value. Taking the same gas phase chemical network used by us in Pety et al. (2007), and assuming a cosmic ionization rate of $\zeta=3 \times 10^{-17} \mathrm{~s}^{-1}$ (Dalgarno 2006), we derive an ionization fraction of $\sim 10^{-8}$ (with respect to $\mathrm{H}$ nuclei) in the densest and coldest regions of the envelope where the $\mathrm{DCO}^{+}$ abundance peaks. This value is, within a factor $\sim 2$, similar to the ionization fraction inferred in prestellar cores such as Barnard 68 (Maret \& Bergin 2007).

\subsubsection{CO outflow parameters}

In the previous section we have estimated the amount of material towards IRAS $22129+7000$ and also evaluated the $\mathrm{CO}$ line emission contribution from the ambient cloud and from the circumstellar envelope (through the optically thin $\mathrm{C}^{18} \mathrm{O}$ line). After subtracting the $\mathrm{CO}$ contribution from the above line-of-sight components we now estimate a CO column density of $\sim 10^{16} \mathrm{~cm}^{-2}$ in the outflow. Taking into account the observed dimensions of the blue- and red-shifted lobes we compute the mass $\left(\sim 0.14 \mathrm{M}_{\odot}\right)$, momentum $\left(\sim 1.0 \mathrm{M}_{\odot} \mathrm{km} \mathrm{s}^{-1}\right)$ and kinetic energy $\left(\sim 6.8 \times 10^{43} \mathrm{erg}\right)$ carried out by the outflow. Note that the mechanical luminosity of the flow $\left(\sim 0.02 \mathrm{~L}_{\odot}\right)$ is still a significant fraction $(\sim 0.4 \%)$ of the radiant luminosity of the driving source. The estimated outflow momentum flux is $\mathrm{F}_{\mathrm{CO}} \sim 3 \times 10^{-5} \mathrm{M}_{\odot} \mathrm{km} \mathrm{s}^{-1} \mathrm{yr}^{-1}$, although the correction factor for inclination effects $\left(\sin i / \cos ^{2} i\right)$ will decrease the actual $\mathrm{F}_{\mathrm{CO}}$ if $i<40^{\circ}$. Hence, $\mathrm{F}_{\mathrm{CO}}$ is intermediate between the observed momentum fluxes found in early Class 0 sources and those found in late Class I sources (e.g, Bontemps et al. 1996, Bachiller 1996). 
TABLE 3

IRAS 22129+700: CO OUTFLOW PARAMETERS

\begin{tabular}{lcccc}
\hline \hline $\begin{array}{l}\text { Outflow } \\
\text { Component }^{a}\end{array}$ & $\begin{array}{c}\text { Mass } \\
\left(\mathrm{M}_{\odot}\right)\end{array}$ & $\begin{array}{c}\text { Momentum } \\
\left(\mathrm{M}_{\odot} \mathrm{km} \mathrm{s}^{-1}\right)\end{array}$ & $\begin{array}{c}\text { Kinetic } \\
\text { Energy } \\
\left(10^{43} \mathrm{erg}\right)\end{array}$ & $\begin{array}{c}\text { Mechanical } \\
\text { Luminosity } \\
\left(10^{-3} \mathrm{~L}_{\odot}\right)\end{array}$ \\
\hline Blueshifted... & 0.06 & 0.4 & 2.9 & 6.9 \\
Redshifted.... & 0.08 & 0.6 & 3.9 & 9.3 \\
Total............ & 0.14 & 1.0 & 6.8 & 16.2 \\
\hline
\end{tabular}

a To calculate the energy and momentum we use an expansion velocity of $7 \mathrm{kms}^{-1}$ and a dynamical age of 35,000 yr (see text).

\section{DISCUSSION AND CONCLUSIONS}

4.1. The Nature of IRAS 22129+7000

As noted earlier IRAS 22129+7000 shows indirect evidence of a protostar/disk system (a collimated outflow) and it shows extended dust emission from the envelope with a large $\mathrm{L}_{s m m} / \mathrm{L}_{\text {bol }}$ ratio $(\simeq 2 \%)$. These characteristics are consistent with the standard observational definition of Class 0 sources (André et al. 2000). Comparison of the derived $\mathrm{L}_{b o l}$ and $\mathrm{M}_{e n v}^{i n}$ values with theoretical evolutionary tracks (Saraceno et al. 1996; André et al. 2000) shows that the source is likely in the interesting Class 0 to I transition. Approximately $50 \%$ of the initial mass envelope must have been accreted at this stage. According to these diagrams, infall must have started in the parental core $\sim(0.5-1) \times 10^{5}$ yr ago. This age is consistent with the dynamical age of the outflow, specially if inclination effects are taken into account.

The peculiar SED of IRAS 22129+7000 shows however a near- and mid-IR excess that produces a high $\mathrm{T}_{b o l}$ of $175 \mathrm{~K}$, consistent with more evolved Class I sources ( 70-650 K; Myers et al. 1998). Given the strong dependence of the short-wavelength IR emission on the viewing angle (Fig.3), the inferred $\mathrm{T}_{b o l}$ is certainly affected by orientation effects. Up-to-date 2D SED models show however that inclination is not enough to reproduce the observed excess. Nevertheless, as mentioned by Robitaille et al. (2006), their large grid of modelled SEDs have some caveats. The most important for our analysis is that none of them includes multiple central sources. An interesting possibility is thus that the source in IRAS $22129+7000$ is a multiple (e.g., binary) stellar system. In fact, prestellar cores can fragment a second time during protostellar collapse and lead to the formation of binary systems (e.g. Duchêne et al. 2007). However, it is not yet clear if the initial conditions in these cores (e.g., the cloud environment) play a major role in such dynamical fragmentation. Simulations predict that external compression waves arriving at the central object at different times or instabilities in the outer accretion disk could be the seed of multiple protostellar systems (e.g., Hennebelle et al. 2003, 2004). In this picture, the presence of both a young and a more evolved YSO sharing a common circumstellar envelope would explain the observed near- and mid-IR excess if the scattered light from the evolved YSO escapes from the outflow cavities.
Higher angular resolution IR observations are required to confirm this scenario. In addition, a larger set of molecular line observations and maps are needed to tightly constrain the physics (infall and rotation velocity fields) and chemistry (molecular content) of this protostar and its environment.

\subsection{Star Formation Near Ced 201 PDR}

IRAS $22129+7000$ is located near Ced 201 PDR $\left(d \sim 0.4 \mathrm{pc}\right.$ and $\left.\mathrm{A}_{V} \sim 1\right)$. Taking into account geometrical dilution and selective dust extinction of FUV dissociating radiation from $\mathrm{BD}+69^{\circ} 1231$, Casey (1991) computed that a region with a radius of at least $\sim 0.6 \mathrm{pc}$ $\left(\sim 300^{\prime \prime}\right)$ around the exciting star is permeated by FUV photons. In particular the FUV radiation field near IRAS $22129+7000$ should be presently at least $\sim 2$ times the mean interstellar radiation field. Aside from dynamical effects associated with the presence of a nearby PDR illuminated by a runaway star (e.g., shock waves), heating and radiation pressure in the region are larger than in other FUV radiation-free environments. Interestingly enough, the $1.2 \mathrm{~mm}$ dust emission (very weak in the PDR itself) peaks again in two dust condensations $\sim 0.25 \mathrm{pc}$ North of Ced 201, roughly in the same direction of outflow red-lobe axis. These cores/clumps have a radius of $\sim 5,000 \mathrm{AU}$ and coincide with an enhancement of the $\mathrm{HCO}^{+} J=1-0$ line emission (see Fig.1). It is very likely that these condensations are being externally heated and compressed by the combined action of the outflow and the PDR associated FUV-driven shock wave. In fact, a similar scenario has presumably led to the formation of IRAS $22129+7000$ itself. We thus suspect that star formation in the B175 globule is triggered by the shocks and radiative feedback induced by $\mathrm{BD}+69^{\circ} 1231$ star and the new generation of low-mass YSOs.

Observations of cores and YSOs provide new insights into how the star formation process is affected by its cloud environment. The vicinity of late type B stars has interesting intermediate properties between isolated dark cores (where FUV-radiation does not play a major role) and cores compressed by the expansion of $\mathrm{H}$ II regions (near more massive stars). Cool PDRs such as Ced 201 are nearly devoid of ionizing photons and thus they provide a very different environment compared to other star forming regions which are photoionized by brighter OB stars. In the special case of Ced 201, the exciting star is moving fast through the region, suggesting that its arrival to the cloud, and thus the triggering, is recent.

We thank P. Hennebelle for useful advice, J. Pety for his help with CLASS90 and the SPECPDR team for their contribution to the project. We also thank the referee for his/her constructive criticisms. JRG was supported by a Marie Curie intra-European Individual Fellowship, contract MEIF-CT-2005-515340. We made use of data products from the 2MASS Catalog, which is a joint project of the U. of Massachusetts and the IPAC/Caltech, funded by NASA and the NSF."

\section{REFERENCES}

Adams, F. C. 1991, ApJ, 382, 544

André, P., Ward-Thompson, D. \& Barsony, M. 1993, ApJ, 406, 122
André, P., Ward-Thompson, D. \& Barsony, M. 2000, Protostars and Planets IV, University of Arizona Press, eds Mannings, V., Boss, A.P., Russell, S. S., p59 
Arce, H,G. \& Sargent, A.I. 2006, ApJ, 646, 1070

Bally, J. \& Reipurth, B. 2001, ApJ, 552, L159

Bachiller, R. 1996, ARA\&A, Volume 34, 111-154

Belloche, A., André, P., Despois, D. \& Blinder, S. 2002, A\&A, 393, 927

Bergin, E.A., \& Tafalla, Mario, 2007, ARA\&A, 45, 339

Berné, O. et al. 2007, A\&A, 469, 575

Bernes, C. 1979, A\&A, 73, 67.

Bontemps, S., André, P., Terebey, S. \& Cabrit, S. 1996, A\&A, 311, 858

Brinch, C., Crapsi, A., Hogerheijde, M. R. \& Jørgensen, J. K. 2007, A\&A, 461, 1037

Cabrit, S. \& Bertout, C. 1986, ApJ, 307, 313

Cabrit, S., Codella, C., Gueth, F., Nisini, B., Gusdorf, A., Dougados, C. \& Bacciotti, F. 2007, A\&A, 468, L2

Casey, S.C. 1991, ApJ, 371, 183

Cernicharo, J. \& Guélin, M. 1987, A\&A, 176, 299

Cesarsky, D., Lequeux, J., Ryter, C., Gerin, M. 2000, A\&A, 354, L87

Dalgarno, A., 2006 Proceedings of the National Academy of Science, vol. 103, Issue 33, 12269-12273

Duchêne, G., Bontemps, S., Bouvier, J., André, P., Djupvik, A. A. \& Ghez, A. M. 2007, A\&A, 476, 229

Flower, D. R. 1999, MNRAS, 305,

Flower, D. R. 2001, JPhB, 305, 651

Furlan E. et al., 2008, to appear in ApJS, astro-ph/07114038.

Goicoechea, J. R. \& Le Bourlot, J. 2007, A\&A, 467, 1

Goicoechea, J. R., Pety, J., Gerin, M., Teyssier, D., Roueff, E., Hily-Blant, P. \& Baek, S. 2006, A\&A, 456, 565

Hennebelle, P., Whitworth, A. P., Gladwin, P. P., \& André, Ph. 2003, MNRAS, 340, 870

Hennebelle, P., Whitworth, A. P., Cha, S.-H., \& Goodwin, S. P. 2004, MNRAS, 348, 687.

Joblin, C. et al. 2005, on Astrochemistry: Recent Successes and Current Challenges, Proceedings of the 231st Symposium of the IAU held in Pacific Grove, California, USA, p.194

Johnstone, D., Wilson, Ch.D., Moriarty-Schieven, G., Joncas, G., Smith, G., Gregersen, E. \& Fich, M. 2000, ApJ, 545, 327
Jørgensen, J.K. et al. 2007, ApJ, 659, 479

Kun, M. 1998, ApJS, 115, 59

Lada, C.J. 1999, The Origin of Stars and Planetary Systems. Edited by Charles J. Lada and Nikolaos D. Kylafis. Kluwer Academic Publishers, 1999, p143

Maret, S, \& Bergin, E.A. 2007, ApJ, 664, 956

Motte, F. \& André, P. 2001, A\&A, 440, 464

Myers, P. C. \& Ladd, E. F. 1993, 413, L4

Myers, P. C., Adams, F. C., Chen, H. \& Schaff, E. 1998, ApJ, 492, 703

Nikolić, S. \& Kun, M. 2004, BaltA, 13, 487

Ossenkopf, V. \& Henning, Th. A\&A, 291, 943

Pety, J., Goicoechea, J. R., Hily-Blant, P., Gerin, M., Teyssier, D. 2007, A\&A, 464, L41

Robitaille, T.P., Whitney, B.A., Indebetouw, R., Wood, K. \& Denzmore, P. 2006, ApJS, 167, 256

Saraceno, P., André, P., Ceccarelli, C., Griffin, M. \& Molinari, S. 1996, A\&A, 309, 827

Shu, F,H., Adams, F.C. \& Lizano, S. 1987, ARA\&A, 25, 23

Skrutskie et al. 2006, AJ, 131, 1163

Smith, J. D. T. et al. 2007, PASP, 119, 1133

Tachihara, K. et al. 2007, ApJ, 659, 1382

Terebey, S., Chandler, C. J. \& André, P. 1993, ApJ, 414, 759

Teyssier, D. \& Sievers, A. 1999. A Fast-Mapping Method for Bolometer Arrary Observations. IRAM technical report.

Tobin, J.J., Looney, L.W., Mundy, L.G., Kwon, W., Hamidouche, M. 2007, ApJ, 659, 1404

Whitney, B,A., Wood, K., Bjorkman, J. E., Cohen, M. 2003, ApJ, 598,1079

Witt, A. N., Graff, S. M., Bohlin, R. C. \& Stecher, T. P. 1987, ApJ, 321,912

Wolf, M. 1908, MNRAS, 69, 117

Zylka, R. 1998, Pocket Cookbook for MOPSIC Software. 